\title{
$\Psi$-pseudodifferential operators and estimates for maximal oscillatory integrals
}

by

\author{
Carlos E. Kenig (Chicago, IL) and Wolfgang Staubach (Edinburgh)
}

\begin{abstract}
We define a class of pseudodifferential operators with symbols $a(x, \xi)$ without any regularity assumptions in the $x$ variable and explore their $L^{p}$ boundedness properties. The results are applied to obtain estimates for certain maximal operators associated with oscillatory singular integrals.
\end{abstract}

1. Introduction. The theory of pseudodifferential operators, as developed by J. J. Kohn and L. Nirenberg [11] and L. Hörmander [8], has played a major role in the analysis of linear partial differential operators. Recall that a pseudodifferential operator is an operator given by

$$
a(x, D) f(x)=\frac{1}{(2 \pi)^{n}} \int_{\mathbb{R}^{n}} a(x, \xi) \widehat{f}(\xi) e^{i\langle x, \xi\rangle} d \xi,
$$

whose symbol $a(x, \xi)$ is assumed to be smooth in both spatial $(x)$ and frequency $(\xi)$ variables and satisfies certain growth conditions. An example is the $S_{o, \delta}^{m}$ symbol class introduced in Hörmander [9], consisting of $a(x, \xi) \in C^{\infty}\left(\mathbb{R}^{n} \times \mathbb{R}^{n}\right)$ with

$$
\left|\partial_{\xi}^{\alpha} \partial_{x}^{\beta} a(x, \xi)\right| \leq C_{\alpha, \beta}\langle\xi\rangle^{m-\varrho|\alpha|+\delta|\beta|},
$$

where $\langle\xi\rangle=\left(1+|\xi|^{2}\right)^{1 / 2}, m \in \mathbb{R}, 0 \leq \delta \leq \varrho \leq 1$.

An important problem in partial differential equations and harmonic analysis is the question of $L^{p}$ boundedness of pseudodifferential operators, which has been extensively studied. Hörmander [9] showed that for $0 \leq \delta<$ $\varrho<1$, operators with symbols in $S_{\varrho, \delta}^{n(\varrho-1) / 2}$ are bounded on $L^{p}$ for $1<p<\infty$. A. Calderón and R. Vaillancourt [1] proved that if $a \in S_{\varrho, \varrho}^{0}$ for $0 \leq \varrho<1$, then the corresponding pseudodifferential operator is bounded on $L^{2}$. In a set of unpublished lecture notes, E. M. Stein showed that if $a(x, \xi) \in S_{\varrho, \delta}^{m}$

2000 Mathematics Subject Classification: Primary 35S05; Secondary 42B20, 42B20.

Key words and phrases: pseudo-pseudodifferential operators, maximal operators, singular oscillatory integrals. 
and either $0 \leq \delta<\varrho=1$ or $0<\delta=\varrho<1$, then $a(x, D)$ is of weak type $(1,1)$ if $m=(\varrho-1) n / 2$. Furthermore, $a(x, D)$ is $L^{p}$ bounded $(1<p<\infty)$ if $(\varrho-1)|1 / 2-1 / p| \geq m / n$ (see also Stein [16]). In [5], C. Fefferman proved that if $a \in S_{\varrho, \delta}^{n(\varrho-1) / 2}$ for $0 \leq \delta<\varrho<1$ then $a(x, D)$ is bounded from $L^{\infty}$ to BMO.

There has also been work on operators with limited regularity and we wish to name only a couple of authors which have inspired us in our investigation. H. Kumano-go [12], Hörmander [10] and C. H. Ching [2] gave examples of bounded symbols which satisfy

$$
\left|\partial_{\xi}^{\alpha} a(x, \xi)\right| \leq C_{\alpha}\langle\xi\rangle^{-\varrho|\alpha|},
$$

with $\varrho \leq 1$, but the corresponding operator is not bounded on $L^{2}$. On the other hand, Nagase [14] was able to show that if $\left|\partial_{\xi}^{\alpha} a(x, \xi)\right| \leq C_{\alpha}\langle\xi\rangle^{-|\alpha|}$, and $\left|\partial_{\xi}^{\alpha} a(x, \xi)-\partial_{\xi}^{\alpha} a(y, \xi)\right| \leq C_{\alpha}|x-y|^{\sigma}\langle\xi\rangle^{-|\alpha|+\sigma \tau}$ for $|\alpha| \leq n+1$, with $0<\sigma \leq 1$ and $0 \leq \tau<1$, then the associated pseudodifferential operator $a(x, D)$ is $L^{2}$ bounded. Later, R. Coifman and Y. Meyer [3] proved that if $a(x, \xi)$ satisfies the first condition of Nagase's theorem and

$$
\left|\partial_{\xi}^{\alpha} a(x, \xi)-\partial_{\xi}^{\alpha} a(y, \xi)\right| \leq C_{\alpha} \omega(|x-y|)\langle\xi\rangle^{-|\alpha|},
$$

where $\omega$ satisfies the condition $\sum_{j=0}^{\infty} \omega\left(2^{-j}\right)^{2}<\infty$, then the $L^{p}$ boundedness holds for $1<p<\infty$.

In this paper we introduce the class of $\Psi$-pseudodifferential operators. The symbols that we are considering satisfy

$$
\left|\partial_{\xi}^{\alpha} a(x, \xi)\right| \leq C_{\alpha}\langle\xi\rangle^{m-\varrho|\alpha|}
$$

for certain values of $m$ and $\varrho$, but have no regularity assumption in the spatial variables $x$. Because of this lack of regularity, the corresponding operators do not have the required pseudo-local property. This means that these operators do not necessarily decrease the singular support when they act on distributions. Hence the name of $\Psi$-pseudodifferential operator is justified since these operators only look like pseudodifferential operators but they are "pseudo-pseudodifferential" operators. We establish the boundedness of these operators in certain $L^{p}$ spaces and we show how they can be used as a tool in proving boundedness results for certain maximal operators in harmonic analysis.

In Section 2, we define $\Psi \Psi$ DOs and discuss their boundedness. In Section 3, we give some applications to estimates for local maximal operators of oscillatory singular integrals of the type considered by S. Wainger [19], I. I. Hirschman [7], C. Fefferman [4], Fefferman and Stein [6] and A. Miyachi [13]. We also prove a theorem similar to P. Sjölin's [15] for oscillatory singular integrals but for the kernels considered in [4]. The proof of Sjölin's result is based on the multidimensional version of Carleson-Hunt's 
theorem, [15], but for the oscillatory singular kernels that we are considering, we can avoid the use of this difficult result by using $\Psi \Psi$ DOs. To some extent, this can be viewed as a related investigation to that of Stein and Wainger [18]. The difference between our and their result is that they establish the $L^{2}$ boundedness of the global maximal operators associated to oscillatory singular integrals with polynomial phases where the degree of the polynomial is larger than or equal to 2 and the kernel is CalderónZygmund, while we prove the $L^{p}$ boundedness $(2 \leq p \leq \infty)$ of local maximal operators associated to oscillatory singular integrals with linear phases and Hirschman-Wainger kernels [7], [19].

The difference between local and global maximal operators is that in the local case the supremum is taken over a compact set but in the global case one takes the supremum over an unbounded open set.

2. $\Psi$-pseudodifferential operators and their $L^{p}$ boundedness. In this section we define the $\Psi$-pseudodifferential operators which are crucial in all our further investigations.

Definition 2.1. Let $a(x, \xi) \in C^{\infty}\left(\mathbb{R}_{\xi}^{n}\right)$ be a measurable function in $x$ and let

$$
\left\|\partial_{\xi}^{\alpha} a(x, \xi)\right\|_{L_{x}^{\infty}} \leq C_{\alpha}\langle\xi\rangle^{m-\varrho|\alpha|}
$$

for some $m \in \mathbb{R}, \varrho \leq 1$ and some constant $C_{\alpha}$. We denote by $L^{\infty} S_{\varrho}^{m}$ the class of symbols with this property.

Given this class of symbols we make the following definition.

Definition 2.2. A $\Psi$-pseudodifferential operator ( $\Psi \Psi \mathrm{DO}$ for short) is an operator $a(x, D)$ which is given by

$$
a(x, D) f=\frac{1}{(2 \pi)^{n}} \int_{\mathbb{R}^{n}} a(x, \xi) \widehat{f}(\xi) e^{i\langle x, \xi\rangle} d \xi, \quad f \in \mathscr{S} .
$$

Thus we do not assume any regularity in the spatial variable $x$ and this will cause singularities for the Schwartz kernel $K(x, y)$ of the operator $a(x, D)$ which might go beyond the set $\left\{(x, x) ; x \in \mathbb{R}^{n}\right\}$. Our main concern, however, is the question of $L^{p}$ boundedness of the $\Psi \Psi$ DOs. To this end we start with the following:

Proposition 2.3. Let $a(x, \xi) \in L^{\infty} S_{o}^{m}, 0 \leq \varrho \leq 1$. Assume that $m<$ $n(\varrho-1) / p$ and $1 \leq p \leq 2$. Then the operator $a(x, D)$ is bounded from $L^{p}$ to $L^{p}$.

Proof. We want to show that

$$
\|a(x, D) u\|_{L^{p}} \lesssim\|u\|_{L^{p}}
$$


Here and below, $a \lesssim b$ means $a \leq C b$ for some constant $C$. We will also denote all generic constants by $C$ even though they might be different from line to line. Our strategy in proving the theorem is to use a Littlewood-Paley decomposition of the symbol $a(x, \xi)$. So let $\left\{\varphi_{k}\right\} \in C_{0}^{\infty}\left(\mathbb{R}^{n}\right)$ be a Littlewood-Paley partition of unity with $\operatorname{supp} \varphi_{0} \subset\{\xi ;|\xi| \leq 2\}$ and $\operatorname{supp} \varphi_{k} \subset$ $\left\{\xi ;|\xi| \sim 2^{k}\right\}$ for $k \geq 1$.

Furthermore, for all multi-indices $\alpha$,

$$
\left|\partial_{\xi}^{\alpha} \varphi_{0}(\xi)\right| \leq c_{\alpha, N}\langle\xi\rangle^{-N} \quad \text { for all } N,
$$

$$
\left|\partial_{\xi}^{\alpha} \varphi_{k}(\xi)\right| \leq c_{\alpha} 2^{-k|\alpha|} \quad \text { for some } c_{\alpha}>0 \text { and } k=1,2, \ldots,
$$

and

$$
\varphi_{0}(\xi)+\sum_{k=1}^{\infty} \varphi_{k}(\xi)=1, \quad \forall \xi .
$$

Using this, we decompose the symbol $a(x, \xi)$ as

$$
a(x, \xi)=a_{0}(x, \xi)+\sum_{k=1}^{\infty} a_{k}(x, \xi)
$$

with $a_{k}(x, \xi)=a(x, \xi) \varphi_{k}(\xi), k=0,1, \ldots$

We shall proceed by estimating the $L^{p}$ norm of each term separately. So the first step is to establish the $L^{p}$ boundedness of

$$
a_{0}(x, D) u(x)=\frac{1}{(2 \pi)^{n}} \int a_{0}(x, \xi) \widehat{u}(\xi) e^{i\langle x, \xi\rangle} d \xi .
$$

By the definition of the symbol class $L^{\infty} S_{\varrho}^{m}$, inequality (2.2), and the Leibniz rule, we realize that

$$
\left|\partial_{\xi}^{\alpha} a_{0}(x, \xi)\right| \leq c_{\alpha, N}\langle\xi\rangle^{-N} \quad \text { for all } N
$$

and all multi-indices $\alpha$. Hence if we look at the Schwartz kernel of $a_{0}(x, D)$

$$
K(x, y)=\frac{1}{(2 \pi)^{n}} \int a_{0}(x, \xi) e^{i\langle x-y, \xi\rangle} d \xi,
$$

and integrate by parts, we obtain, for all integers $M>0$,

$$
|K(x, y)| \lesssim\langle x-y\rangle^{-2 M} \int\left|\left(1-\Delta_{\xi}\right)^{M} a_{0}(x, \xi)\right| d \xi .
$$

Using (2.7) and choosing $M>n / 2$, we see that both integrals $\int|K(x, y)| d x$ and $\int|K(x, y)| d y$ are convergent and Schur's lemma yields

$$
\left\|a_{0}(x, D) u\right\|_{L^{p}} \lesssim\|u\|_{L^{p}}
$$

Now let us analyze $a_{k}(x, D) u(x)=(2 \pi)^{-n} \int a_{k}(x, \xi) \widehat{u}(\xi) e^{i\langle x, \xi\rangle} d \xi$ for $k \geq 1$. Using the definition of $L^{\infty} S_{\varrho}^{m}$, inequality (2.3) and the Leibniz rule we realize that

$$
\left|\partial_{\xi}^{\alpha} a_{k}(x, \xi)\right| \leq c_{\alpha} 2^{k(m-\varrho|\alpha|)} \quad \text { for some } c_{\alpha}>0 \text { and } k=1,2, \ldots
$$


where we have also used the assumption $\varrho \leq 1$. We note that $a_{k}(x, D) u(x)$ can be written as

$$
a_{k}(x, D) u(x)=\int K_{k}(x, y) u(x-y) d y
$$

with

$$
K_{k}(x, y)=\frac{1}{(2 \pi)^{n}} \int a_{k}(x, \xi) e^{i\langle y, \xi\rangle} d \xi=\check{a}_{k}(x, y),
$$

where $\check{a}_{k}$ denotes the inverse Fourier transform of $a_{k}(x, \xi)$ with respect to $\xi$. One observes that

$$
\begin{aligned}
\left\|a_{k}(x, D) u\right\|_{L^{p}}^{p} & =\int\left|\int K_{k}(x, y) u(x-y) d y\right|^{p} d x \\
& =\int\left|\int K_{k}(x, y) \sigma_{k}(y) \frac{1}{\sigma_{k}(y)} u(x-y) d y\right|^{p} d x,
\end{aligned}
$$

with weight functions $\sigma_{k}(y)$ which will be chosen in a moment. Therefore, Hölder's inequality yields

$$
\begin{aligned}
& \left\|a_{k}(x, D) u\right\|_{L^{p}}^{p} \\
& \quad \leq \int\left\{\int\left|K_{k}(x, y)\right|^{p^{\prime}}\left|\sigma_{k}(y)\right|^{p^{\prime}} d y\right\}^{p / p^{\prime}}\left\{\int \frac{|u(x-y)|^{p}}{\left|\sigma_{k}(y)\right|^{p}} d y\right\} d x
\end{aligned}
$$

where $1 / p+1 / p^{\prime}=1$. Now for an $l>n / p$, we define a class of weight functions by setting

$$
\sigma_{k}(y)= \begin{cases}2^{-k \varrho n / p}, & |y| \leq 2^{-k \varrho} \\ 2^{-k \varrho(n / p-l)}|y|^{l}, & |y|>2^{-k \varrho} .\end{cases}
$$

By Hausdorff-Young's theorem and the estimate (2.11), first for $\alpha=0$ and then for $|\alpha|=l$, we have

$$
\begin{aligned}
\int 2^{-k p^{\prime} \varrho n / p}\left|K_{k}(x, y)\right|^{p^{\prime}} d y & \leq 2^{-k p^{\prime} \varrho n / p}\left\{\int\left|a_{k}(x, \xi)\right|^{p} d \xi\right\}^{p^{\prime} / p} \\
& \lesssim 2^{-k p^{\prime} \varrho n / p}\left\{\int_{|\xi| \sim 2^{k}} 2^{p m k} d \xi\right\}^{p^{\prime} / p} \\
& \lesssim 2^{k p^{\prime}(m-n(\varrho-1) / p)}
\end{aligned}
$$

and

$$
\begin{aligned}
& \int 2^{-k \varrho p^{\prime}(n / p-l)}\left|K_{k}(x, y)\right|^{p^{\prime}}|y|^{p^{\prime} l} d y \\
& \lesssim 2^{-k \varrho p^{\prime}(n / p-l)}\left\{\int\left|\nabla_{\xi}^{l} a_{k}(x, \xi)\right|^{p} d \xi\right\}^{p^{\prime} / p} \\
& \lesssim 2^{-k \varrho p^{\prime}(n / p-l)}\left\{\int_{|\xi| \sim 2^{k}} 2^{k p(m-\varrho l)} d \xi\right\}^{p^{\prime} / p} \lesssim 2^{k p^{\prime}(m-n(\varrho-1) / p)}
\end{aligned}
$$


Hence, splitting the integral into $|y| \leq 2^{-k \varrho}$ and $|y|>2^{-k \varrho}$ yields

$$
\begin{aligned}
\left\{\int\left|K_{k}(x, y)\right|^{p^{\prime}}\left|\sigma_{k}(y)\right|^{p^{\prime}} d y\right\}^{p / p^{\prime}} & \lesssim\left\{2^{k p^{\prime}(m-n(\varrho-1) / p)}\right\}^{p / p^{\prime}} \\
& =2^{k p(m-n(\varrho-1) / p)} .
\end{aligned}
$$

Furthermore if we choose $l>n / p$ then

$$
\int \frac{d y}{\left|\sigma_{k}(y)\right|^{p}}=2^{k \varrho n} \int_{|y| \leq 2^{-k \varrho}} d y+2^{k \varrho p(n / p-l)} \int_{|y|>2^{-k \varrho}}|y|^{-p l} d y=C(n),
$$

a constant that only depends on the dimension $n$. Thus (2.14) yields

$$
\left\|a_{k}(x, D) u\right\|_{L^{p}} \lesssim 2^{k(m-n(\varrho-1) / p)}\|u\|_{L^{p}} .
$$

Summing up and using the bounds for $a_{0}(x, D)$ and $a_{k}(x, D)$, we obtain

$$
\begin{aligned}
\|a(x, D) u\|_{L^{p}} & \leq\left\|a_{0}(x, D) u\right\|_{L^{p}}+\sum_{k=1}^{\infty}\left\|a_{k}(x, D) u\right\|_{L^{p}} \\
& \lesssim\|u\|_{L^{p}}+\sum_{k=1}^{\infty} 2^{k(m-n(\varrho-1) / p)}\|u\|_{L^{p}}
\end{aligned}
$$

We observe that the series above converges if $m<n(\varrho-1) / p$. This ends the proof of the proposition.

REMARK 2.4. If $0 \leq \varrho \leq 1$ then the $L^{2}$ boundedness result is sharp. In fact, following the example of Hörmander [10] one can see that if $m \geq$ $n(\varrho-1) / 2$ then there are symbols $a(x, \xi)$ in the Hörmander class $S_{\varrho, 1}^{m}$ whose corresponding Kohn-Nirenberg quantization is not bounded on $L^{2}$. Since obviously $S_{\varrho, 1}^{m} \subset L^{\infty} S_{\varrho}^{m}$, it follows at once that our $L^{2}$ result is sharp.

The condition $m<n(\varrho-1) / 2$ also guarantees $L^{\infty}$ boundedness. More precisely, we have

Proposition 2.5. Let $a(x, \xi) \in L^{\infty} S_{\varrho}^{m}$. Assume that $m<n(\varrho-1) / 2$ and $0 \leq \varrho \leq 1$. Then the operator $a(x, D)$ is bounded from $L^{\infty}$ to $L^{\infty}$.

Proof. We will use the same technique as in the proof of Proposition 2.3. Observing that for $u \in L^{\infty}$,

$$
\left\|a_{k}(x, D) u\right\|_{L^{\infty}} \leq\|u\|_{L^{\infty}} \int\left|K_{k}(x, y)\right| d y
$$

one only needs to estimate $\int\left|K_{k}(x, y)\right| d y$. We split the integral into

$$
\begin{aligned}
\int\left|K_{k}(x, y)\right| d y & =\int_{\substack{|y| \leq 2^{-k \varrho}\\
}}\left|K_{k}(x, y)\right| d y+\int_{|y|>2^{-k \varrho}}\left|K_{k}(x, y)\right| d y \\
& =: I_{1}+I_{2} .
\end{aligned}
$$


To estimate $I_{1}$ we use the Cauchy-Schwarz inequality and (2.17) for the case $p=p^{\prime}=2$. Hence

$$
I_{1} \leq\left\{\int_{|y| \leq 2^{-k \varrho}} d y\right\}^{1 / 2}\left\{\int\left|K_{k}(x, y)\right|^{2} d y\right\}^{1 / 2} \lesssim 2^{k(m-n(\varrho-1) / 2)} .
$$

To estimate $I_{2}$ we use again the Cauchy-Schwarz inequality and (2.18) for the case $p=p^{\prime}=2$. This yields

$$
\begin{aligned}
I_{2} & \leq\left\{\int_{|y|>2^{-k \varrho}}|y|^{-2 l} d y\right\}^{1 / 2}\left\{\int\left|K_{k}(x, y)\right|^{2}|y|^{2 l} d y\right\}^{1 / 2} \\
& \lesssim 2^{k(m-n(\varrho-1) / 2)} .
\end{aligned}
$$

Thus

$$
\left\|a_{k}(x, D) u\right\|_{L^{\infty}} \lesssim 2^{k(m-n(\varrho-1) / 2)}\|u\|_{L^{\infty}}
$$

for $k=1,2, \ldots$. Summing up and using the hypothesis on $m$ and the bounds for $a_{0}(x, D)$ and $a_{k}(x, D)$, we obtain

$$
\|a(x, D) u\|_{L^{\infty}} \lesssim\|u\|_{L^{\infty}}+\sum_{k=1}^{\infty} 2^{k(m-n(\varrho-1) / 2)}\|u\|_{L^{\infty}} \lesssim\|u\|_{L^{\infty}}
$$

as desired.

Remark 2.6. For $0 \leq \varrho \leq 1$ the $L^{\infty}$ result in Proposition 2.5 is sharp. In fact, if we consider the symbol $a(\xi)=\varphi(\xi)|\xi|^{m} e^{i|\xi|^{1-\varrho}} \in L^{\infty} S_{\varrho}^{m}$, where $\varphi=0$ near zero and $\varphi=1$ for large $\xi$, then it is known that the operator associated to this symbol does not map $L^{\infty}$ to $L^{\infty}$ if $m \geq n(\varrho-1) / 2$ (see e.g. Miyachi [13]).

A consequence of the previous propositions is

TheOREm 2.7. Let $a(x, \xi) \in L^{\infty} S_{\varrho}^{m}, 0 \leq \varrho \leq 1$. Then if $m<n(\varrho-1) / 2$ then $a(x, D)$ is a bounded operator from $L^{p}$ to $L^{p}$ for all $p \in[2, \infty]$. For $\varrho=1$ and $m<0$ the range of $p$ for which the operator is $L^{p}$ bounded is $[1, \infty]$.

Proof. This follows by interpolating the $L^{2}$ result of Proposition 2.3 with the $L^{\infty}$ result of Proposition 2.5. The last claim follows from the interpolation between the $L^{1}$ result of Proposition 2.3 and $L^{\infty}$ boundedness result of Proposition 2.5, which are valid for $m<0$ and $\varrho=1$.

We conclude this section by noting that the number of derivatives needed in the $\xi$ variables need not be infinite. Indeed, by following our proofs one can reduce the number of derivatives to a finite number depending on the dimension $n$. 
3. Applications to maximal operator estimates. In this section we consider certain maximal functions associated with strongly singular integral operators and oscillatory integrals. In each case we can establish boundedness in $L^{p}$ for the local maximal operators.

Let us first consider singular integrals that are given by convolution with the distribution on $\mathbb{R}^{n}$ that away from the origin agrees with the function

$$
K^{a, b}(x)=\frac{e^{i|x|^{a}}}{|x|^{n+b}} \varphi(x)
$$

with $b \in \mathbb{R}, a \in(-\infty, 0] \cup(1, \infty)$ and $\varphi$ a radial smooth compactly supported function, equal to 1 in the unit ball. Consider the strongly singular integral operator $T f(x)=K^{a, b}(x) * f(x)$. This operator is bounded on $L^{p}$, provided that $a<0, b<-a n / 2,1<p<\infty$ and $|1 / 2-1 / p| \leq(a n / 2+b) / a n$ (see Hirschman [7], Wainger [19], Stein [17], C. Fefferman [4] and Miyachi [13]). Setting

$$
\beta=\frac{a n / 2+b}{a-1} \quad \text { and } \quad K_{t}^{a, b}(x)=\frac{1}{t^{n-\beta}} K^{a, b}\left(\frac{x}{t}\right),
$$

we are interested in the $L^{p}$ estimates for the maximal operator

$$
T_{*} f(x):=\sup _{0<t<1}\left|\left(K_{t}^{a, b} * f\right)(x)\right| .
$$

Using the Fourier transform, one can write $T_{*}$ as

$$
T_{*} f(x):=\frac{1}{(2 \pi)^{n}} \sup _{0<t<1}\left|\int\left(K_{t}^{a, b}\right)^{\wedge}(\xi) \widehat{f}(\xi) e^{i\langle x, \xi\rangle} d \xi\right| .
$$

Since

$$
\left(K^{a, b}\right)^{\wedge}(\xi)=\frac{e^{i|\xi|^{\alpha}}}{|\xi|^{\beta}} \theta(\xi) \quad \text { with } \quad \alpha=\frac{a}{a-1}, \beta=\frac{a n / 2+b}{a-1}
$$

and $\theta(\xi)$ is a smooth function on $\mathbb{R}^{n}$, which vanishes near zero, and equals 1 outside a bounded set (this is essentially the case), the Fourier transform of $K_{t}^{a, b}$ is

$$
\left(K_{t}^{a, b}\right)^{\wedge}(\xi)=\frac{e^{i|t \xi|^{\alpha}}}{|\xi|^{\beta}} \theta(t \xi) .
$$

Therefore to estimate $T_{*}$ it is enough to estimate

$$
\int \frac{e^{i|t(x) \xi|^{\alpha}}}{|\xi|^{\beta}} \theta(t(x) \xi) \widehat{f}(\xi) e^{i\langle x, \xi\rangle} d \xi
$$

for an arbitrary measurable function $t(x) \in[0,1]$. So we are dealing with estimates for a $\Psi \Psi D O$ with symbol

$$
\frac{e^{i|t(x) \xi|^{\alpha}}}{|\xi|^{\beta}} \theta(t(x) \xi) \in L^{\infty} S_{1-\alpha}^{-\beta}=L^{\infty} S_{1 /(1-a)}^{-(a n / 2+b) /(a-1)} .
$$

Thus we have the following 


\section{THEOREM 3.1.}

(i) If $a>1$ and $b>a(n / p-n / 2)$ or $a<0$ and $b<a(n / p-n / 2)$ then $T_{*}: L^{p} \rightarrow L^{p}$ is bounded for $1 \leq p \leq 2$.

(ii) If $a>1$ and $b>0$ or $a<0$ and $b<0$ then $T_{*}: L^{p} \rightarrow L^{p}$ is bounded for $2 \leq p \leq \infty$.

Proof. (i) follows from Proposition 2.3 and (ii) follows from Theorem 2.7. Next let

$$
T_{\lambda}^{a, b}(f)(x)=\int_{\mathbb{R}^{n}} e^{i\langle\lambda, y\rangle} K^{a, b}(y) f(x-y) d y,
$$

with $\lambda \in \mathbb{R}^{n}$. It was shown by Sjölin [15] that for an appropriate CalderónZygmund kernel $K$, the operator

$$
C f(x):=\sup _{\lambda \in \mathbb{R}^{n}}\left|\int_{\mathbb{R}^{n}} e^{i\langle\lambda, y\rangle} K(y) f(x-y) d y\right|
$$

is bounded in $L^{2}\left(\mathbb{R}^{n}\right)$.

For $l<1$ we define the maximal operator $T^{*} f(x):=\sup _{|\lambda|<l}\left|T_{\lambda} f(x)\right|$. Then we have

Theorem 3.2. $T^{*}$ is bounded from $L^{p}\left(\mathbb{R}^{n}\right) \rightarrow L^{p}\left(\mathbb{R}^{n}\right)$ for $2 \leq p \leq \infty$, provided $a>1, b>0$.

Proof. Once again, after linearization and using the Fourier transform, we are led to study a $\Psi \Psi \mathrm{DO}$

$$
T(x, D) f(x)=\frac{1}{(2 \pi)^{n}} \int_{\mathbb{R}^{n}} \frac{e^{i|\xi-\lambda(x)|^{\alpha}}}{|\xi-\lambda(x)|^{\beta}} \theta(\xi-\lambda(x)) e^{i\langle x, \xi\rangle} \widehat{f}(\xi) d \xi
$$

where $\lambda(x)$ is an arbitrary measurable function with $|\lambda(x)|<l<1$, and $\alpha$, $\beta, \theta$ as defined previously. A calculation shows that

$$
\left|\partial_{\xi}^{\gamma}\left\{\frac{e^{i|\xi-\lambda(x)|^{\alpha}}}{|\xi-\lambda(x)|^{\beta}} \theta(\xi-\lambda(x))\right\}\right| \leq C_{\gamma, l}\langle\xi\rangle^{\frac{a n / 2+b}{1-a}-\frac{|\gamma|}{1-a}} \quad \text { for }|\lambda|<l .
$$

Now to prove the claim, apply Theorem 2.7.

\section{References}

[1] A. P. Calderón and R. Vaillancourt, A class of bounded pseudo-differential operators, Proc. Nat. Acad. Sci. U.S.A. 6 (1972), 1185-1187.

[2] C. H. Ching, Pseudo-differential operators with nonregular symbols, J. Differential Equations 11 (1972), 436-447.

[3] R. Coifman et Y. Meyer, Au-delà des opérateurs pseudo-différentiels, Astérisque 57 (1978).

[4] C. Fefferman, Inequalities for strongly singular convolution operators, Acta Math. 124 (1970), 9-36. 
[5] C. Fefferman, $L^{p}$ bounds for pseudo-differential operators, Israel J. Math. 14 (1973), 413-417.

[6] C. Fefferman and E. M. Stein, $H^{p}$ spaces of several variables, Acta Math. 129 (1972), 137-193.

[7] I.I. Hirschman, On multiplier transformations, Duke Math. J. 26 (1959), 221-242.

[8] L. Hörmander, Pseudo-differential operators, Comm. Pure Appl. Math. 18 (1965), 501-517.

[9] —, Pseudo-differential operators and hypoelliptic equations, in: Singular Integrals (Chicago, IL, 1966), Proc. Sympos. Pure Math. 10, Amer. Math. Soc., Providence, RI, 1967, 138-183.

[10] - On the $L^{2}$ continuity of pseudo-differential operators, Comm. Pure Appl. Math. 24 (1971), 529-535.

[11] J. J. Kohn and L. Nirenberg, An algebra of pseudo-differential operators, ibid. 18 (1965), 269-305.

[12] H. Kumano-go, A problem of Nirenberg on pseudo-differential operators, ibid. 23 (1970), 115-121.

[13] A. Miyachi, On some singular Fourier multipliers, J. Fac. Sci. Univ. Tokyo Sect. IA Math. 28 (1981), 267-315.

[14] M. Nagase, The $L^{p}$-boundedness of pseudo-differential operators with non-regular symbols, Comm. Partial Differential Equations 2 (1977), 1045-1061.

[15] P. Sjölin, Convergence almost everywhere of certain singular integrals and multiple Fourier series, Ark. Mat. 9 (1971), 65-90.

[16] E. M. Stein, Harmonic Analysis: Real-Variable Methods, Orthogonality, and Oscillatory Integrals, Princeton Math. Ser. 43, Princeton Univ. Press, Princeton, NJ, 1993.

[17] —, Singular integrals, harmonic functions, and differentiability properties of functions of several variables, in: Singular Integrals (Chicago, IL, 1966), Proc. Sympos. Pure Math. 10, Amer. Math. Soc., Providence, RI, 1967, 316-335.

[18] E. M. Stein and S. Wainger, Oscillatory integrals related to Carleson's theorem, Math. Res. Lett. 8 (2001), 789-800.

[19] S. Wainger, Special trigonometric series in k-dimensions, Mem. Amer. Math. Soc. 59 (1965).

Department of Mathematics

University of Chicago

5734 South University Avenue

Chicago, IL 60637, U.S.A.

E-mail: cek@math.uchicago.edu
Department of Mathematics Heriot-Watt University Colin Maclaurin Building

Edinburgh, EH14 4AS, UK E-mail: W.Staubach@hw.ac.uk

Received September 24, 2006

Revised version October 17, 2007 